\title{
Efficacy and safety of COX-2 inhibitors for advanced non-small-cell lung cancer with chemotherapy: a meta-analysis
}

This article was published in the following Dove Press journal:

OncoTargets and Therapy

\author{
Ping Dai \\ Jing Li \\ Xiao-Ping $\mathrm{Ma}$ \\ Jian Huang \\ Juan-Juan Meng \\ Ping Gong
}

Department of Oncology, The First Affiliated Hospital, Shihezi University School of Medicine, Shihezi, Xinjiang, People's Republic of China
Correspondence: Ping Gong Department of Oncology, The First Affiliated Hospital, Shihezi University School of Medicine, No. 107, North Second Road, Shihezi, Xinjiang 832000, People's Republic of China Email gongpI I22@sina.com
Background: The study of cyclooxygenase-2 (COX-2) inhibitors is now mired in controversy. We performed a meta-analysis to assess the efficacy and safety profile of COX-2 inhibitors in patients with advanced non-small-cell lung cancer (NSCLC).

Patients and methods: A literature search of PubMed, EMBASE, the Cochrane Central databases, and ClinicalTrials.gov, up until March 26, 2017, identified relevant randomized controlled trials. Data analysis was performed using Stata 12.0.

Results: Six eligible trials (1,794 patients) were selected from the 407 studies that were identified initially. A significant difference, favoring COX-2 inhibitors plus chemotherapy over chemotherapy alone, was observed in the overall response rate (relative risk $[R R]=1.25$, 95\% confidence interval [CI]: 1.06-1.48). Further, we conducted two subgroup analyses according to the type of COX-2 inhibitors (celecoxib, rofecoxib, or apricoxib) and treatment line (first or second chemotherapy). The first-line treatment includes: NP (changchun red bean + cisplatin or carboplatin), GP (double fluorine cytidine + cisplatin or carboplatin), or TP (paclitaxel + cisplatin or carboplatin, docetaxel + cisplatin or carboplatin). The second-line treatment includes two internationally recognized compounds, one is docetaxel and the other is the pemetrexed, both of which are individually selected. In subgroup analysis, significantly increased overall response rate $(\mathrm{ORR})$ results were found for rofecoxib plus chemotherapy $(\mathrm{RR}=1.56,95 \% \mathrm{CI}$ : 1.08-2.25) and COX-2 inhibitor given with first-line chemotherapy ( $\mathrm{RR}=1.27,95 \% \mathrm{CI}$ : 1.07-1.50). However, there was no difference between COX-2 inhibitors plus chemotherapy and chemotherapy alone in overall survival (hazard ratio [HR] $=1.04,95 \%$ CI: $0.91-1.18$ ), progression-free survival $(\mathrm{HR}=0.97,95 \% \mathrm{CI}: 0.86-1.10)$, and 1 -year survival rate $(\mathrm{RR}=1.03$, 95\% CI: 0.89-1.20). Toxicity did not differ significantly between COX-2 inhibitors plus chemotherapy and chemotherapy alone with the exception of leukopenia ( $R R=1.21,95 \% \mathrm{CI}$ : 1.03-1.42), thrombocytopenia ( $\mathrm{RR}=1.32,95 \% \mathrm{CI}: 1.04-1.67)$, and cardiovascular events $(\mathrm{RR}=2.39,95 \% \mathrm{CI}: 1.06-5.42)$. The results of the Egger's test indicated no significant difference in primary outcomes.

Conclusion: COX-2 inhibitors improved ORR of advanced NSCLC with chemotherapy, but had no effect on survival indices. Moreover, COX-2 inhibitors may lead to higher rates of hematologic toxicities and cardiovascular events.

Keywords: cyclooxygenase-2 inhibitors, non-small-cell lung cancer, chemotherapy, overall survival, meta-analysis

\section{Introduction}

A growing number of preclinical studies showed that overexpression of cyclooxygenase-2 (COX-2) had been implicated as a tumor-initiating and tumor-promoting event for several common solid tumors, including lung, breast, and colon cancers. ${ }^{1-3}$ 
Approximately $70 \%$ of adenocarcinomas in non-small-cell lung cancer (NSCLC) have been found to exhibit increased COX-2 expression. ${ }^{4} \mathrm{COX}-2$ expression in tumors appears to be instrumental in tumor resistance to apoptosis, angiogenesis, invasion, and immune suppression. ${ }^{5}$ Further, selective COX-2 inhibitors have been shown to inhibit the growth of lung cancer cell lines and to enhance the effectiveness of selected chemotherapy against NSCLC cell lines in xenograft models. ${ }^{6}$ These studies ${ }^{5,7}$ suggest nonsteroidal anti-inflammatory drugs (NSAIDs) may act on multiple tumor-progression targets via both COX-2-dependent and -independent pathways. Based on these observations, COX-2 inhibitors have been evaluated in combination with chemotherapy for the management of metastatic NSCLC in patients who have failed prior chemotherapy. However, current clinical trials on the benefit of COX-2 inhibitors in cancer treatment report conflicting results. Indeed, some studies ${ }^{2,4,6}$ demonstrated that COX-2 inhibitors could enhance antitumor activity of conventional anticancer agents in vitro and in vivo. However, many studies have confirmed that COX-2 inhibitors did not appear to enhance efficacy or improve patient-reported symptoms and can also lead to certain toxicity. ${ }^{8,9}$

There are three meta-analyses ${ }^{10-12}$ about the efficacy and safety profile of COX-2 inhibitors that have been published. All the three studies reported that COX-2 inhibitors could increase overall response rate (ORR) in patients with advanced NSCLC. Of these, two studies ${ }^{10,11}$ indicated that celecoxib significantly increased risk of hematologic toxicities, while Chen et $\mathrm{al}^{12}$ reported that COX-2 inhibitors plus chemotherapy was associated with a higher incidence of cardiovascular events compared with chemotherapy alone. Two meta-analyses ${ }^{10,12}$ did not carry out a hazard ratio (HR) analysis of outcome indicators overall survival (OS) and progression-free survival (PFS). While conducting meta-analysis, Hou et $\mathrm{al}^{10}$ and Chen et $\mathrm{al}^{12}$ only focused on celecoxib. Moreover, Hou et $\mathrm{al}^{10}$ included six studies with 1,181 patients, describing all end points without subgroup analysis. To better assess the efficacy and safety profile of COX-2 inhibitors combined with chemotherapy for patients with advanced NSCLC, the meta-analysis of data from published randomized controlled trials (RCTs) in this field was performed.

\section{Materials and methods}

\section{Literature search strategy}

This meta-analysis was reported according to Preferred Reporting Items for Systematic Reviews and Meta-Analyses guidelines. ${ }^{13}$ Systematic computerized searches of PubMed,
Embase, and Cochrane data bases for reports dated up to March 26, 2017 were performed with the following search terms: "cyclooxygenase-2 inhibitor", "COX-2 inhibitor", "non-small-cell lung cancer", "NSCLC", "chemotherapy". All reference lists from the trials selected by electronic searching were scanned to further identify relevant trials. Ethical approval was not required for this study.

\section{Literature selection and exclusion}

The following criteria were used for study selection: 1) the RCTs compared the efficacy and safety profile of adding COX-2 inhibitors to chemotherapy alone; 2) only including patients with cytologically or histologically confirmed NSCLC stage IIIB or IV; 3) full paper in English language was published; and 4) studies needed to have measured at least one of the following outcomes as their end points: OS, PFS, 1-year survival rate (SR), ORR, and toxicities.

If a study was a duplicate or the study's data could not be extracted or obtained through contact with the author, it was excluded from our analysis.

\section{Data extraction}

The final articles included were independently assessed by two authors. In the case of disagreement, another author was consulted to resolve the dispute, and a final decision was made by majority vote. The relevant information included study design, patient characteristics, interventions, controls, and outcomes. For some missing survival indices such as OS and PFS, HR and 95\% confidence interval (CI) were extracted from the survival curve. ${ }^{14}$ Regarding toxicity, we considered both hematological (leukopenia, thrombocytopenia, and anemia) and nonhematological (nausea/vomiting, diarrhea, asthenia, and cardiotoxicity) grade 3 and grade 4 effects of treatment.

\section{Quality assessment of included studies}

Two investigators independently evaluated the methodological quality of eligible trials using the Cochrane collaboration tool for assessing risk of bias ${ }^{15}$ (random sequence generation, allocation concealment, blinding of participants and personnel, blinding of outcome assessment, incomplete outcome data, selective reporting, and other sources of bias).

\section{Statistical analysis}

Dichotomous data, including 1-year SR, ORR, and toxicities, were compared with a pooled risk ratio (RR) with a 95\% CI. Survival indices of OS and PFS were expressed as HR with a 95\% CI. ${ }^{16,17}$ This meta-analysis was performed using Stata 
12.0. Heterogeneity between studies was also analyzed using chi-square tests, with the significance level set to $P<0.1 .^{18}$ No heterogeneity was observed when $I^{2}=0 \%$. However, when $I^{2}>50 \%$, studies were considered to have significant heterogeneity. If the data were homogeneous under a fixedeffects model, the type of COX-2 inhibitors and treatment line were identified as key sources of heterogeneity in the main outcomes (OS, PFS, 1-year SR, and ORR). ${ }^{18}$ Heterogeneity was then dealt with using subgroups based on these modifiers. If the data were still heterogeneous, we introduced a random-effects model. Whereas when $I^{2}<50 \%$, a fixedeffects model was used instead. ${ }^{18}$

A funnel plot was used to estimate potential publication bias, with an asymmetric plot suggesting possible bias. ${ }^{19}$ In the funnel plot, larger studies that provide a more precise estimate of an interventions effect form the spout of the funnel, whereas smaller studies with less precision form the cone end of the funnel. Finally, the Egger's test was employed to address quantitative detection bias. ${ }^{20}$

\section{Results}

\section{Characteristics of individual studies}

We identified 407 publications from the electronic databases (Figure 1), of which 86 were excluded as duplicates and
273 were excluded based on selection criteria. This resulted in 48 articles, which were independently read by two authors. Eventually, six studies ${ }^{21-26}$ involving 1,794 patients were included in our meta-analysis. The characteristics of each individual study are presented in Table 1 .

\section{Quality of the included studies}

The risk of bias in the included studies was strictly evaluated. Four studies ${ }^{23-26}$ describe a random component in the sequence generation process and the concealment of treatment allocation, and the four trials ${ }^{23-26}$ were designed as double-blind trials. In addition, one study ${ }^{22}$ lost large amounts of data, which may lend to a certain attrition bias. Details of methodological approch are presented in Table 2.

\section{Overall survival}

All studies ${ }^{21-26}$ including 1,794 patients reported HR for OS. When assessing the effect on OS (as shown in Figure 2), COX-2 inhibitors plus chemotherapy did not significantly differ from chemotherapy alone $(\mathrm{HR}=1.04$, 95\% CI: $\left.0.91-1.18, I^{2}=0.0 \%, P=0.808\right)$. Further, we conducted two subgroup analyses according to the type of

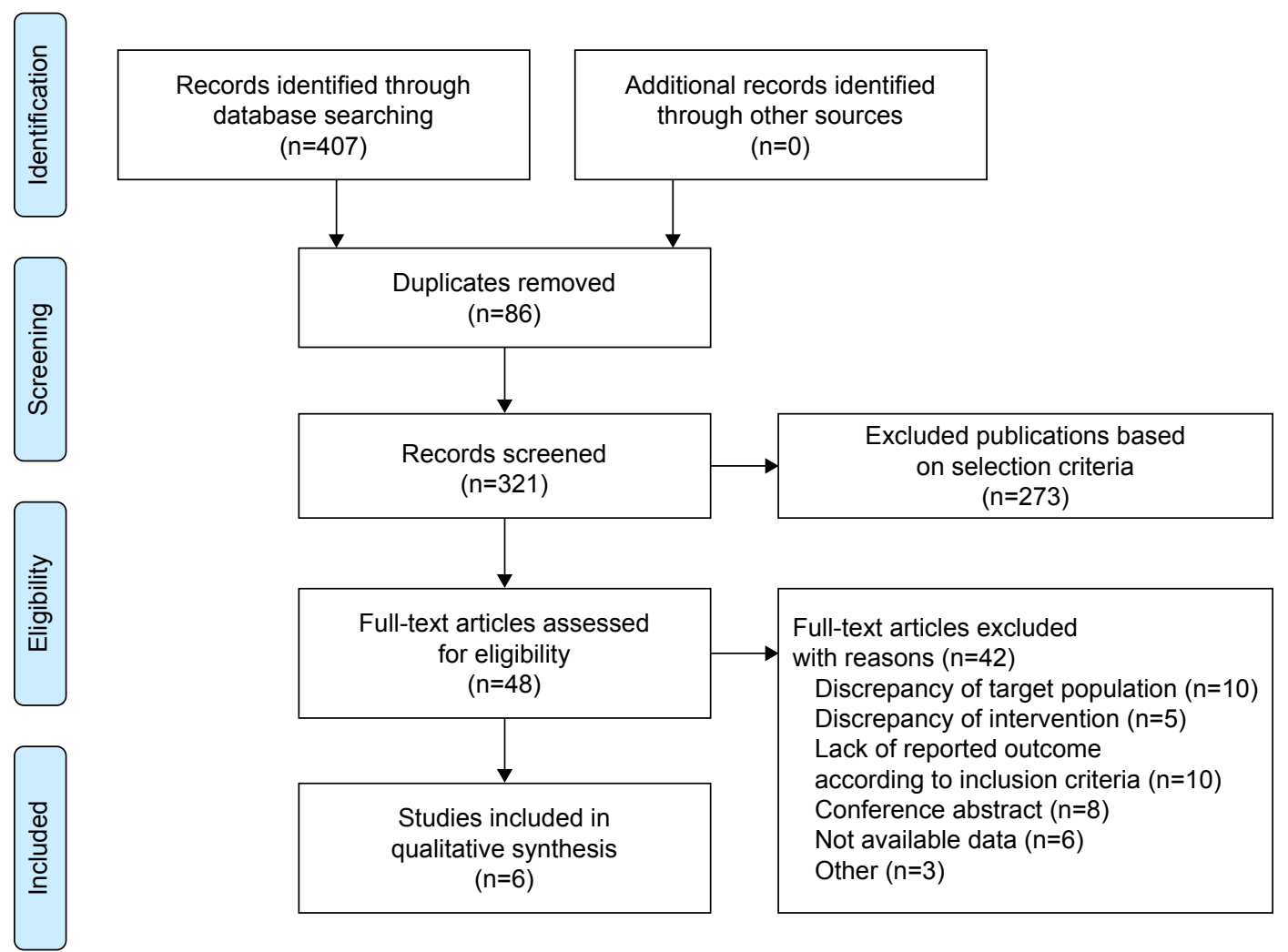

Figure I Summary of trial identification and selection. 
Table I The characteristics of each individual study

\begin{tabular}{|c|c|c|c|c|c|c|c|c|c|c|}
\hline \multirow[t]{2}{*}{ Study } & \multirow[t]{2}{*}{ Year } & \multirow[t]{2}{*}{ Phase } & \multirow{2}{*}{$\begin{array}{l}\text { Study } \\
\text { period }\end{array}$} & \multirow[t]{2}{*}{ Country } & \multirow{2}{*}{$\begin{array}{l}\text { Sample } \\
\text { (I/C) }\end{array}$} & \multirow{2}{*}{$\begin{array}{l}\text { Age (years) } \\
\text { (I/C) }\end{array}$} & \multirow{2}{*}{$\begin{array}{l}\text { Male } \\
\text { (female) } \\
(\mathrm{I} / \mathrm{C})\end{array}$} & \multicolumn{3}{|l|}{ Histology, I/C } \\
\hline & & & & & & & & Adenocarcinoma & $\begin{array}{l}\text { Squamous cell } \\
\text { carcinoma }\end{array}$ & Other \\
\hline $\begin{array}{l}\text { Lilenbaum } \\
\text { et } \mathrm{al}^{21}\end{array}$ & 2006 & II & $\begin{array}{l}\text { February } \\
2002 \text { to } \\
\text { September } \\
2003\end{array}$ & USA & $133(67 / 66)$ & $\begin{array}{l}62.7(37-84) / \\
63.5(4 I-78)\end{array}$ & $\begin{array}{l}40(27) / \\
40(26)\end{array}$ & NA & NA & NA \\
\hline $\begin{array}{l}\text { Gridelli } \\
\text { et a }\left.\right|^{22}\end{array}$ & 2007 & III & $\begin{array}{l}\text { January } 2003 \\
\text { to May } 2005\end{array}$ & Italy & $400(|49 / 25|)$ & $\begin{array}{l}61.5(29-71) / \\
59(37-70)\end{array}$ & $\begin{array}{l}\text { I20 (29)/ } \\
202(49)\end{array}$ & $68 / 134$ & $47 / 53$ & $34 / 64$ \\
\hline $\begin{array}{l}\text { Koch } \\
\text { et } \mathrm{al}^{23}\end{array}$ & 2011 & III & $\begin{array}{l}\text { May } 2003 \text { to } \\
\text { May } 2006\end{array}$ & Sweden & $316(158 / 158)$ & $\begin{array}{l}66(38-85) / \\
65(37-85)\end{array}$ & $\begin{array}{l}73(85) / \\
87(7 I)\end{array}$ & $77 / 94$ & $38 / 27$ & $43 / 36$ \\
\hline $\begin{array}{l}\text { Groen } \\
\text { et } \mathrm{al}^{24}\end{array}$ & 2011 & III & $\begin{array}{l}\text { July } 2003 \text { to } \\
\text { December } \\
2007\end{array}$ & $\begin{array}{l}\text { the } \\
\text { Netherlands }\end{array}$ & $56 I(28 I / 280)$ & $\begin{array}{l}62(40-84) / \\
61(33-84)\end{array}$ & $\begin{array}{l}\text { I84 (97)/ } \\
|7|(109)\end{array}$ & $138 / 132$ & $44 / 57$ & 99/91 \\
\hline $\begin{array}{l}\text { Edelman } \\
\text { et } \mathrm{a}^{25}\end{array}$ & 2015 & II & NA & USA & $72(36 / 36)$ & $62 / 66$ & $\begin{array}{l}20(16) / \\
20(16)\end{array}$ & $24 / 25$ & $8 / 6$ & $4 / 5$ \\
\hline $\begin{array}{l}\text { Edelman } \\
\text { et } \mathrm{al}^{26}\end{array}$ & 2017 & III & $\begin{array}{l}\text { November } \\
2013 \text { to } \\
\text { January } 2016\end{array}$ & USA & $312(154 / 158)$ & $\begin{array}{l}64(38-83) / \\
64(36-89)\end{array}$ & $\begin{array}{l}82(72) / \\
87(7 I)\end{array}$ & NA & $44 / 43$ & NA \\
\hline
\end{tabular}

Note: Data are presented as mean (range) unless otherwise specified.

Abbreviations: I/C, interventions/control; NA, not applicable.

COX-2 inhibitors (celecoxib, rofecoxib, or apricoxib) and treatment line (first or second). Unfortunately, no clinical benefit in OS was found among the groups: celecoxib ( $\left.\mathrm{HR}=1.05,95 \% \mathrm{CI}: 0.90-1.22, I^{2}=0.0 \%, P=0.532\right)$, rofecoxib $\left(\mathrm{HR}=1.00,95 \% \mathrm{CI}: 0.75-1.34, I^{2}=\right.$ not applicable [NA], $P=\mathrm{NA}$ ), apricoxib (HR $=1.04,95 \% \mathrm{CI}: 0.64-1.69$, $\left.I^{2}=\mathrm{NA}, P=\mathrm{NA}\right)$, first-line treatment $(\mathrm{HR}=1.01,95 \%$ CI: $\left.0.88-1.16, I^{2}=0.0 \%, P=0.819\right)$, and second-line treatment $\left(\mathrm{HR}=1.19,95 \% \mathrm{CI}: 0.88-1.60, I^{2}=0.0 \%\right.$, $P=0.508)$.

\section{Progression-free survival}

All studies ${ }^{21-26}$ including 1,794 patients reported HR for PFS. We also assessed the effect on PFS (summarized in Figure 3), and found that COX-2 inhibitors plus chemotherapy did not significantly differ from chemotherapy alone $(\mathrm{HR}=0.97$,

Table 2 The risk of bias in the included studies

\begin{tabular}{|c|c|c|c|c|c|c|c|c|c|}
\hline Study & Year & Country & $\begin{array}{l}\text { Random } \\
\text { sequence } \\
\text { generation }\end{array}$ & $\begin{array}{l}\text { Allocation } \\
\text { concealment }\end{array}$ & $\begin{array}{l}\text { Blinding of } \\
\text { participants } \\
\text { and personnel }\end{array}$ & $\begin{array}{l}\text { Blinding of } \\
\text { outcome } \\
\text { assessment }\end{array}$ & $\begin{array}{l}\text { Incomplete } \\
\text { outcome } \\
\text { data }\end{array}$ & $\begin{array}{l}\text { Selective } \\
\text { reporting }\end{array}$ & Other bias \\
\hline $\begin{array}{l}\text { Lilenbaum } \\
\text { et } \mathrm{al}^{2 !}\end{array}$ & 2006 & USA & Not reported & Not reported & Not reported & $\begin{array}{l}\text { Not } \\
\text { reported }\end{array}$ & Intent to treat & $\begin{array}{l}\text { Not } \\
\text { reported }\end{array}$ & $\begin{array}{l}\text { No other } \\
\text { source of bias }\end{array}$ \\
\hline $\begin{array}{l}\text { Gridelli } \\
\text { et } \mathrm{al}^{22}\end{array}$ & 2007 & Italy & Not reported & Not reported & Not reported & $\begin{array}{l}\text { Not } \\
\text { reported }\end{array}$ & Intent to treat & $\begin{array}{l}\text { Not } \\
\text { reported }\end{array}$ & $\begin{array}{l}\text { No other } \\
\text { source of bias }\end{array}$ \\
\hline $\begin{array}{l}\text { Koch } \\
\text { et } \mathrm{al}^{23}\end{array}$ & 2011 & Sweden & Minimization & $\begin{array}{l}\text { Central } \\
\text { allocation }\end{array}$ & Yes & $\begin{array}{l}\text { Not } \\
\text { reported }\end{array}$ & Intent to treat & $\begin{array}{l}\text { Not } \\
\text { reported }\end{array}$ & $\begin{array}{l}\text { No other } \\
\text { source of bias }\end{array}$ \\
\hline $\begin{array}{l}\text { Groen } \\
\text { et } \mathrm{al}^{24}\end{array}$ & 2011 & $\begin{array}{l}\text { the } \\
\text { Netherlands }\end{array}$ & Centralized & Not reported & Not reported & Yes & Intent to treat & $\begin{array}{l}\text { Not } \\
\text { reported }\end{array}$ & $\begin{array}{l}\text { No other } \\
\text { source of bias }\end{array}$ \\
\hline $\begin{array}{l}\text { Edelman } \\
\text { et } \mathrm{al}^{25}\end{array}$ & 2015 & USA & Centralized & $\begin{array}{l}\text { Central } \\
\text { allocation }\end{array}$ & Yes & $\begin{array}{l}\text { Not } \\
\text { reported }\end{array}$ & Intent to treat & $\begin{array}{l}\text { Not } \\
\text { reported }\end{array}$ & $\begin{array}{l}\text { No other } \\
\text { source of bias }\end{array}$ \\
\hline $\begin{array}{l}\text { Edelman } \\
\text { et } \mathrm{al}^{26}\end{array}$ & 2017 & USA & $\begin{array}{l}\text { Stratified random- } \\
\text { permuted-blocks } \\
\text { procedure }\end{array}$ & $\begin{array}{l}\text { Central } \\
\text { allocation }\end{array}$ & Yes & $\begin{array}{l}\text { Not } \\
\text { reported }\end{array}$ & Intent to treat & $\begin{array}{l}\text { Not } \\
\text { reported }\end{array}$ & $\begin{array}{l}\text { No other } \\
\text { source of bias }\end{array}$ \\
\hline
\end{tabular}

Notes: *The dose of chemotherapeutic agents was not mentioned in the trial; **the dose of carboplatin was not mentioned in the trial.

Abbreviations: bid, twice daily; CBP, carboplatin; d, day; DDP, cisplatin; DTX, docetaxel; iv, intravenously; ECOG PS, Eastern Cooperative Oncology Group performance status; GEM, gemcitabine; ivgtt, intravenous drip; PCl, prolonged constant infusion; NVB, vinorelbine; PET, pemetrexed; po, orally; q, every; w, weeks. 


\begin{tabular}{|c|c|c|c|c|c|}
\hline $\begin{array}{l}\text { Extent of } \\
\text { disease, stage }\end{array}$ & $\begin{array}{l}\text { ECOG } \\
\text { PS }\end{array}$ & $\begin{array}{l}\text { Treatment } \\
\text { line }\end{array}$ & Interventions & Control & $\begin{array}{l}\text { Follow-up } \\
\text { (months) }\end{array}$ \\
\hline IIIB, IV & $\begin{array}{l}\text { ECOG } \\
0-1\end{array}$ & Second & $\begin{array}{l}\text { Celecoxib } 400 \mathrm{mg} \text { po bid + DTX } \\
35 \mathrm{mg} \mathrm{m}^{-2} \text { or GEM I,000 mg m }{ }^{-2}+\text { CPT-II } \\
60-100 \mathrm{mg} \mathrm{m}^{-2} \text { ivgtt day I and } 8 \text { day, q3w }\end{array}$ & $\begin{array}{l}\text { DTX } 35 \mathrm{mg} \mathrm{m}^{-2} \text { or GEM } \\
\mathrm{I}, 000 \mathrm{mg} \mathrm{m} \mathrm{m}^{-2}+\text { CPT-II } 60-100 \mathrm{mg} \mathrm{m}^{-2} \\
\text { ivgtt day I and } 8 \text { day, q3w }\end{array}$ & 19 \\
\hline IIIB, IV & $\begin{array}{l}\text { ECOG } \\
0-1\end{array}$ & First & $\begin{array}{l}\text { Rofecoxib } 50 \mathrm{mg} \text { po qd + GEM I,200 } \mathrm{mg} \mathrm{m}^{-2} \\
\text { in } 30 \text {-minute or PCI GEM I,200 mg m}{ }^{-2} \\
\text { over } 120 \text {-minute iv infusions days I and } 8+ \\
\text { DDP } 80 \mathrm{mg} \mathrm{m}^{-2} \text { ivgtt qd day I, q3w }\end{array}$ & $\begin{array}{l}\text { GEM I,200 } \mathrm{mg} \mathrm{m}^{-2} \text { in } 30 \text {-minute or PCl } \\
\text { GEM I,200 } \mathrm{mg} \mathrm{m}^{-2} \text { over I20-minute iv } \\
\text { infusions days I and } 8 \text { + DDP } 80 \mathrm{mg} \mathrm{m}^{-2} \\
\text { ivgtt qd day I, q3w }\end{array}$ & 28 \\
\hline IIIB, IV & $\begin{array}{l}\text { ECOG } \\
0-2\end{array}$ & First & $\begin{array}{l}\text { Celecoxib } 400 \mathrm{mg} \text { po bid + GEM or NVB + } \\
\text { CBP or DDP, ivgtt q3 } w^{*}\end{array}$ & $\begin{array}{l}\text { Placebo + GEM or NVB + CBP or } \\
\text { DDP, ivgtt } q 3 w\end{array}$ & 36 \\
\hline IIIB, IV & $\begin{array}{l}\text { ECOG } \\
0-2\end{array}$ & First & $\begin{array}{l}\text { Celecoxib } 400 \text { mg po bid + DTX } 75 \text { mg m-2 } \\
\text { ivgtt qd day I + CBP ivgtt qd day I, q3w** }\end{array}$ & $\begin{array}{l}\text { Placebo + DTX } 75 \mathrm{mg} \mathrm{m}^{-2} \text { ivgtt qd } \\
\text { day I + CBP ivgtt qd day I, q3w }\end{array}$ & 53 \\
\hline IIIB, IV & $\begin{array}{l}\text { ECOG } \\
0-2\end{array}$ & Second & $\begin{array}{l}\text { Apricoxib } 400 \mathrm{mg} \text { po qd + DTX } 75 \mathrm{mg} \mathrm{m}^{-2} \\
\text { or PET } 500 \mathrm{mg} \mathrm{m}^{-2} \text {, q3w }\end{array}$ & $\begin{array}{l}\text { Placebo } 400 \mathrm{mg} \text { po qd DTX } 75 \mathrm{mg} \mathrm{m}^{-2} \\
\text { or PET } 500 \mathrm{mg} \mathrm{m}^{-2} \text {, q3w }\end{array}$ & NA \\
\hline IIIB, IV & $\begin{array}{l}\text { ECOG } \\
0-2\end{array}$ & First & $\begin{array}{l}\text { Celecoxib } 400 \mathrm{mg} \text { po bid + } \\
\text { CBP + PET } 500 \mathrm{mg} \mathrm{m}^{-2} \text { day I, q3w for } \\
\text { nonsquamous or celecoxib } 400 \mathrm{mg} \text { po bid + } \\
\text { CBP day I+ GEM I,000 } \mathrm{mg} \mathrm{m}^{-2} \text { day I and } \\
\text { day } 8 \text {, q3w for squamous }\end{array}$ & $\begin{array}{l}\text { Placebo }+ \text { CBP }+ \text { PET } 500 \mathrm{mg} \mathrm{m}^{-2} \\
\text { day I, q3w for nonsquamous or } \\
\text { placebo }+ \text { CBP day I + GEM } \\
\mathrm{I}, 000 \mathrm{mg} \mathrm{m}^{-2} \text { day I and day } 8, \mathrm{q} 3 \mathrm{w} \text { for } \\
\text { squamous }\end{array}$ & 31 \\
\hline
\end{tabular}

95\% CI: $\left.0.86-1.10, I^{2}=0.0 \%, P=0.849\right)$. As previously mentioned, we also performed two subgroup analyses. However, no significant differences were obtained in the following groups: celecoxib ( $\mathrm{HR}=0.96,95 \% \mathrm{CI}$ : 0.83-1.12, $\left.P^{2}=0.0 \%, P=0.584\right)$, rofecoxib $(\mathrm{HR}=1.00,95 \% \mathrm{CI}: 0.76-1.31$, $\left.I^{2}=\mathrm{NA}, P=\mathrm{NA}\right)$, apricoxib $(\mathrm{HR}=0.97,95 \% \mathrm{CI}: 0.58-$ 1.62, $\left.I^{2}=\mathrm{NA}, P=\mathrm{NA}\right)$, first-line treatment $(\mathrm{HR}=0.97,95 \%$ CI: $\left.0.84-1.11, I^{2}=0.0 \%, P=0.578\right)$, or second-line treatment $\left(\mathrm{HR}=0.99,95 \% \mathrm{CI}: 0.74-1.33, I^{2}=0.0 \%, P=0.924\right)$.

\section{One-year survival rate}

Five RCTs including 1,482 patients reported 1-year mortality rate figures. We next evaluated the effect on 1-year SR (summarized in Figure 4). COX-2 inhibitors plus chemotherapy did not significantly differ from chemotherapy alone $\left(\mathrm{RR}=1.03,95 \% \mathrm{CI}: 0.89-1.20, I^{2}=0.0 \%, P=0.531\right)$. Moreover, when grouped by the type of COX-2 inhibitors, subgroup analysis also did not yield significant results: celecoxib $\left(\mathrm{RR}=1.03,95 \% \mathrm{CI}: 0.86-1.22, I^{2}=36.3 \%\right.$, $P=0.208)$, rofecoxib $(\mathrm{RR}=1.06,95 \% \mathrm{CI}: 0.78-1.44$, $\left.I^{2}=\mathrm{NA}, P=\mathrm{NA}\right)$, or apricoxib $(\mathrm{RR}=1.00,95 \% \mathrm{CI}: 0.15-6.72$, $\left.I^{2}=\mathrm{NA}, P=\mathrm{NA}\right)$. Similar results were found in the subgroup analysis according to treatment line: first-line treatment $\left(\mathrm{RR}=1.08,95 \% \mathrm{CI}: 0.92-1.27, I^{2}=0.0 \%, P=0.958\right)$ and second-line treatment $(\mathrm{RR}=0.68,95 \% \mathrm{CI}: 0.41-1.14$, $\left.I^{2}=0.0 \%, P=0.676\right)$.

\section{Overall response rate}

Four RCTs including 1,410 patients reported ORR. When evaluating the effect on ORR (summarized in Figure 5), COX-2 inhibitors combined with chemotherapy were found to be more effective than chemotherapy alone $(\mathrm{RR}=1.25$, 95\% CI: $\left.1.06-1.48, I^{2}=0.0 \%, P=0.420\right)$. To better assess the efficacy of COX-2 inhibitors for advanced NSCLC, we also conducted further subgroup analysis. Significantly increased ORRs were observed for rofecoxib $(\mathrm{RR}=1.56$, 95\% CI: $\left.1.08-2.25, I^{2}=\mathrm{NA}, P=\mathrm{NA}\right)$ and first-line treatment $\left(\mathrm{RR}=1.27,95 \% \mathrm{CI}: 1.07-1.50, I^{2}=0.0 \%, P=0.451\right)$. Whereas celecoxib $\left(\mathrm{RR}=1.18,95 \%\right.$ CI: $0.98-1.42, I^{2}=0.0 \%$, $P=0.562$ ) and second-line treatment with COX-2 inhibitors for patients with advanced NSCLC showed no significant difference $(\mathrm{RR}=0.49,95 \% \mathrm{CI}$ : 0.09-2.60, $\left.I^{2}=\mathrm{NA}, P=\mathrm{NA}\right)$.

\section{Toxicities}

Finally, we assessed the toxicities of COX-2 inhibitors plus chemotherapy for patients with advanced NSCLC. Results indicated that grade 3 and grade 4 toxicities of leukopenia, 


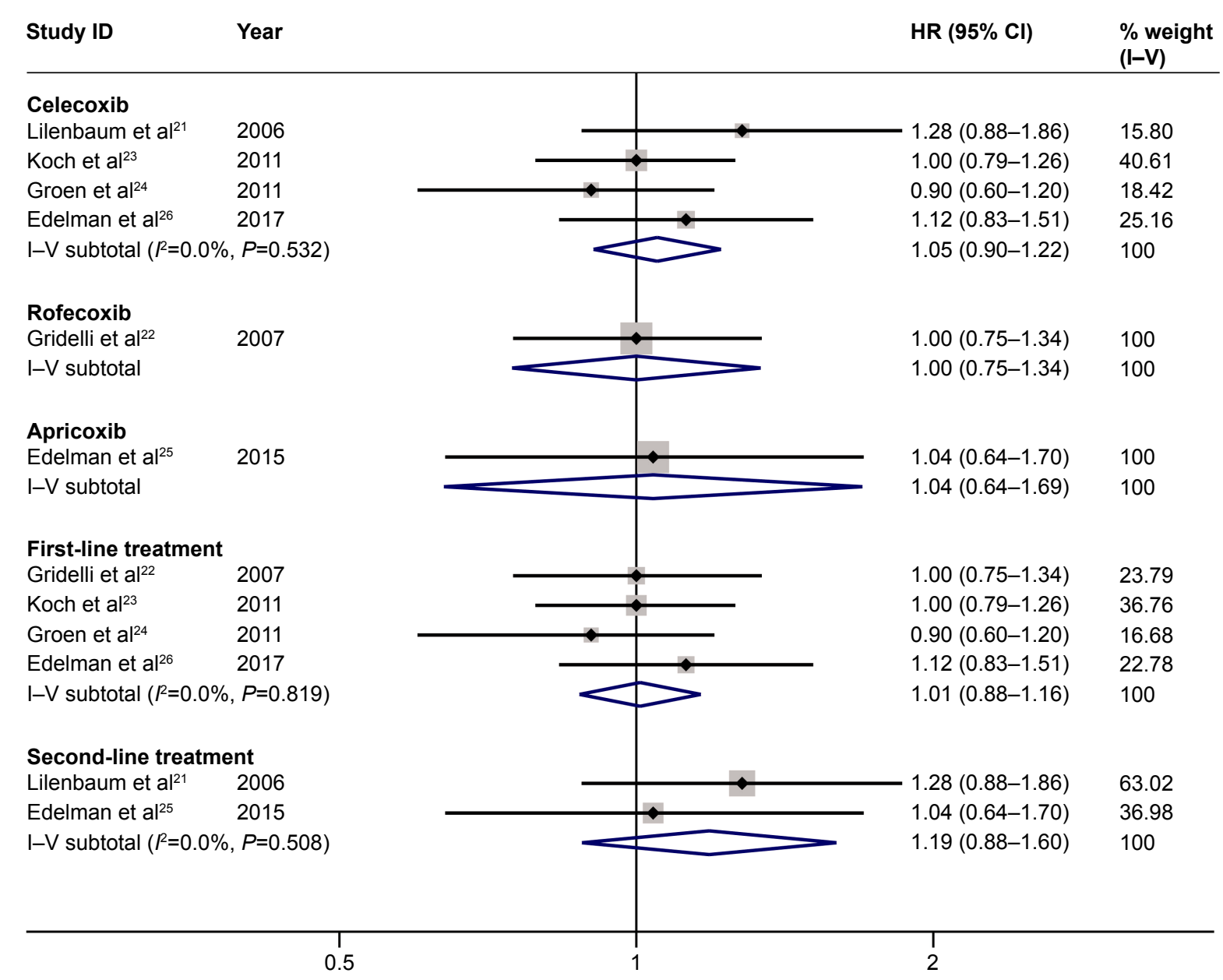

Figure 2 Forest plot of overall survival from subgroup analysis.

Abbreviations: $\mathrm{HR}$, hazard ratio; $\mathrm{I}-\mathrm{V}$, inverse variance.

thrombocytopenia, and cardiovascular events increased with the addition of COX-2 inhibitors: leukopenia $(\mathrm{RR}=1.21$, 95\% CI: $\left.1.03-1.42, I^{2}=0.0 \%, P=0.499\right)$, thrombocytopenia (RR $=1.32,95 \%$ CI: $\left.1.04-1.67, I^{2}=0.0 \%, P=0.560\right)$, and cardiotoxicity $\left(\mathrm{RR}=2.39,95 \% \mathrm{CI}: 1.06-5.42, I^{2}=0.0 \%\right.$, $P=0.690$ ). However, significantly increased risks of other toxicities (anemia, nausea/vomiting, diarrhea, asthenia) and grade 3 and grade 4 effects of treatment were not found. Detailed data are listed in Table 3.

\section{Publication bias}

No publication bias was observed for any of the outcomes based on the symmetry of the funnel plots. Furthermore, the results of the Egger's test indicated no significant difference in primary outcomes: OS (bias $=0.708,95 \%$ CI: -3.086 to $4.051, P=0.632$ ), PFS (bias $=-0.387,95 \%$ CI: -4.508 to $3.733, P=0.807$ ), ORR ( bias $=-0.835$, 95\% CI: -6.033 to $4.363, P=0.561$ ), and 1 -year SR (bias $=-0.940,95 \%$ CI: $-3.748-1.869, P=0.365$ ).

\section{Discussion}

In this meta-analysis, we evaluated six clinical trials that included 1,794 advanced NSCLC patients. Our metaanalysis indicated a significantly increased ORR with COX-2 inhibitors plus chemotherapy over chemotherapy alone. COX-2 is reported to interfere with angiogenesis, apoptosis, and tumor invasiveness. ${ }^{27}$ Increased expression of COX-2 has been found in lung cancer and has been associated with worse prognosis. ${ }^{28,29} \mathrm{COX}-2$ inhibitors inhibit the growth of human lung cancer cells as single agents as well as in combination with chemotherapy. Subgroup analysis reported that rofecoxib rather than celecoxib may produce a significantly increased ORR of advanced NSCLC with chemotherapy. Zhou et $\mathrm{al}^{11}$ found that both celecoxib and rofecoxib can improve the ORR of advanced NSCLC with chemotherapy. Inconsistencies in these results may be due to a different sample size. The celecoxib plus chemotherapy subgroup of Zhou et al contained six RCTs, whereas this study included four RCTs. In addition, Zhou et al and this meta-analysis 


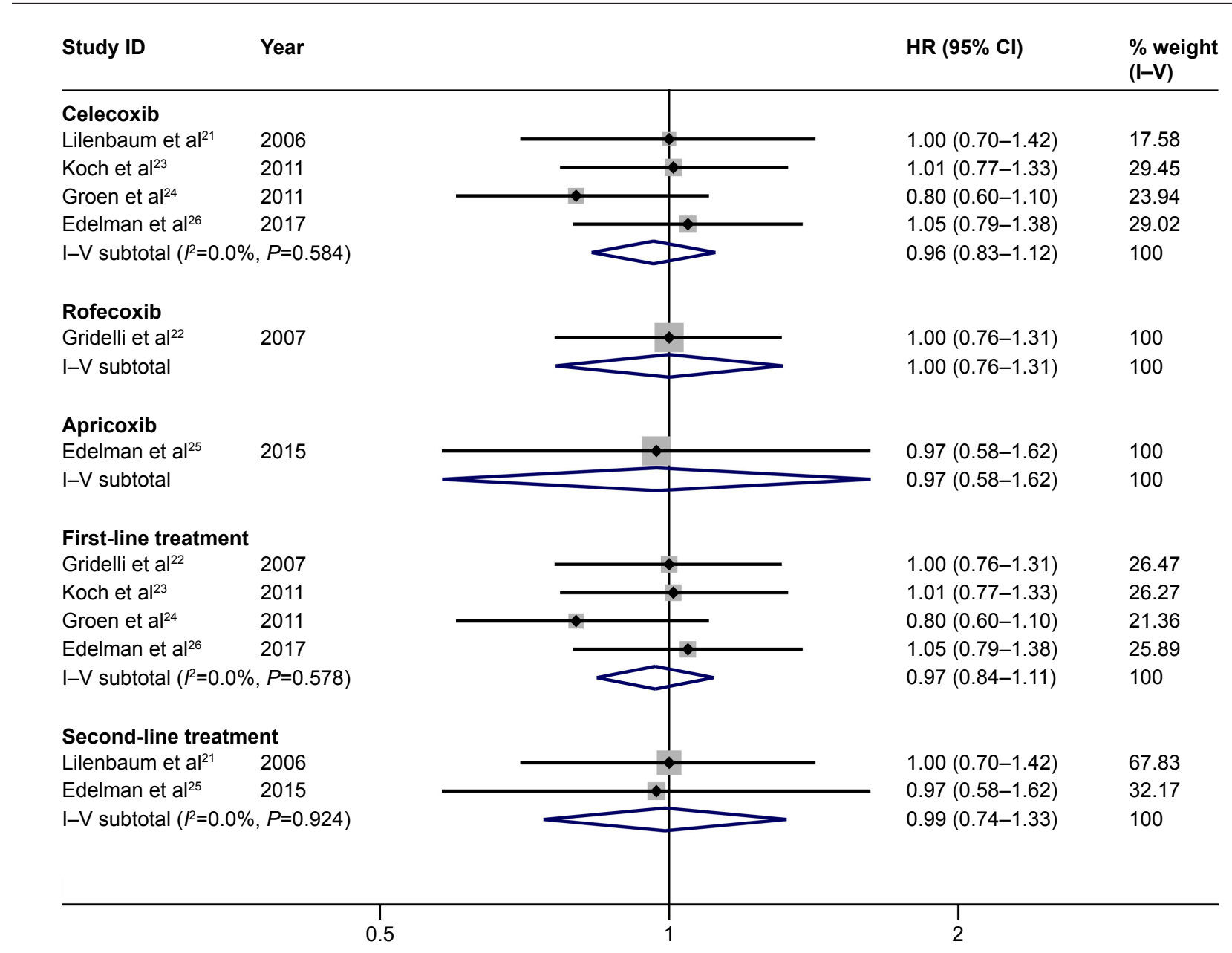

Figure 3 Forest plot of progression-free survival from subgroup analysis.

Abbreviations: $H R$, hazard ratio; $I-V$, inverse variance.

included only one trial on rofecoxib, and so the reliability of the results may be reduced and further research with a large sample is needed to confirm these results. According to treatment line, we observed a statistically significant favorable effect of first-line chemotherapy with COX-2 inhibitors on ORR but no change in second-line chemotherapy. Since there was only one study (by Lilenbaum et $\mathrm{al}^{21}$ ) which included COX-2 inhibitors as second-line chemotherapy, more research is needed to verify this conclusion. However, there was no significant difference found in 1-year SR of advanced NSCLC between COX-2 inhibitors plus chemotherapy and chemotherapy alone. In the subgroup analysis that was based on the type of COX-2 inhibitors and treatment line, 1-year SR also did not change between COX-2 inhibitors plus chemotherapy and chemotherapy alone. Similar results were obtained for OS and PFS. In all subgroup analyses, COX-2 inhibitors plus chemotherapy showed no significant influence on OS and PFS compared with chemotherapy alone. Three studies $^{10-12}$ reported results consistent with this study, where
COX-2 inhibitors plus chemotherapy had no advantage over 1-year SR compared to chemotherapy alone. Only Zhou et $\mathrm{a}^{11}$ calculated pooled HR of OS and PFS, and indicated that difference in OS and PFS durations of patients on COX-2 inhibitors plus chemotherapy and chemotherapy alone was not statistically significant. There has been no research to report that COX-2 inhibitors plus chemotherapy can reduce mortality of patients with advanced NSCLC. Therefore, further study on how to improve the 1-year SR, OS, or PFS of patients with advanced NSCLC is still necessary. The abovementioned results showed that COX-2 inhibitors may increase ORR of chemotherapy with advanced NSCLC, especially combined with first-line treatment. However, no similar change was found in the survival indices.

Toxicities were graded according to Common Terminology Criteria for Adverse Events v3.0 of the National Cancer Institute. ${ }^{30}$ This meta-analysis included both hematological and nonhematological grade 3 and grade 4 side effects of treatment. A higher frequency of leukopenia, thrombocytopenia, 


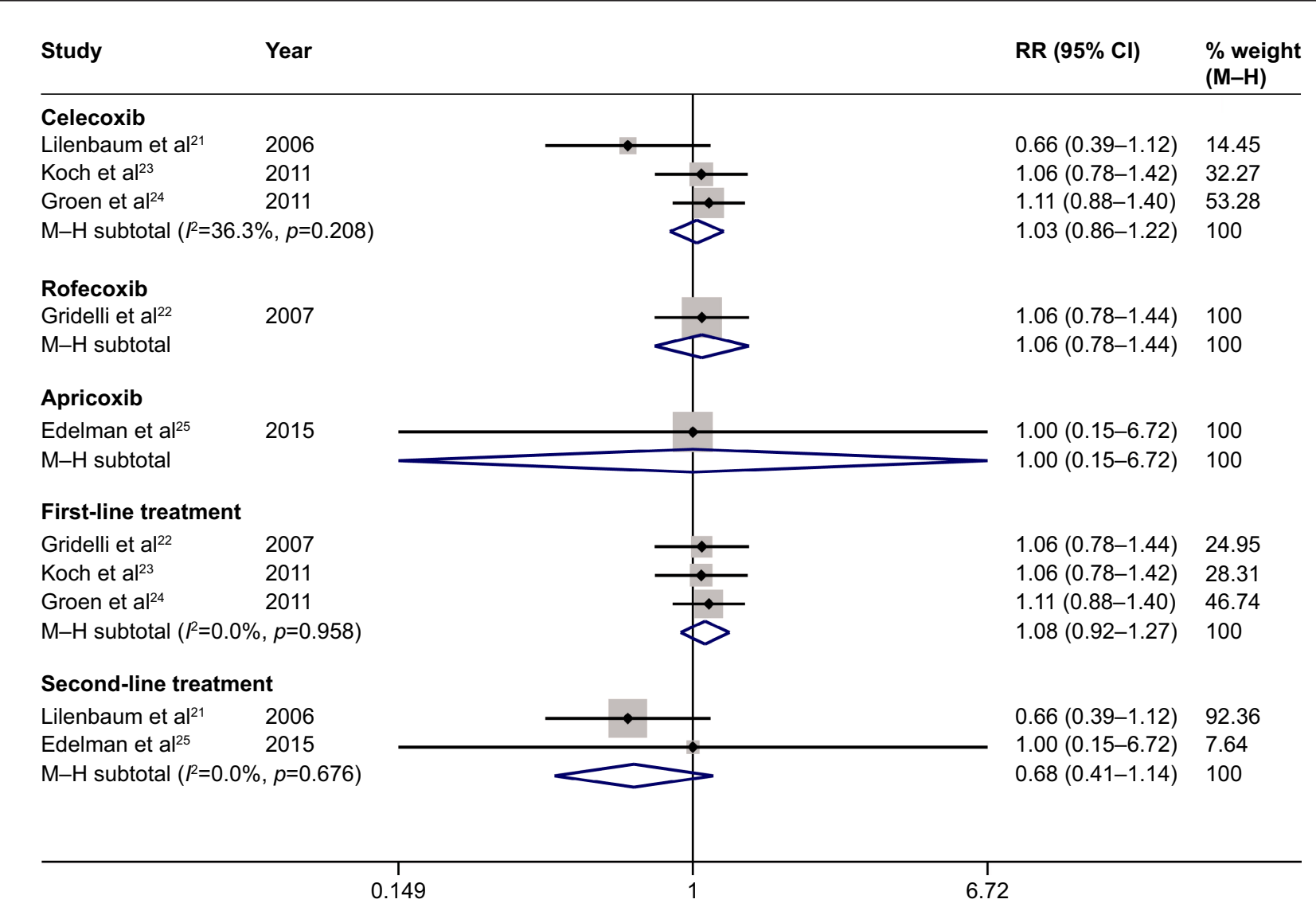

Figure 4 Forest plot of I-year survival rate from subgroup analysis.

Abbreviations: RR, risk ratio; $\mathrm{M}-\mathrm{H}$, Mantel-Haenszel.

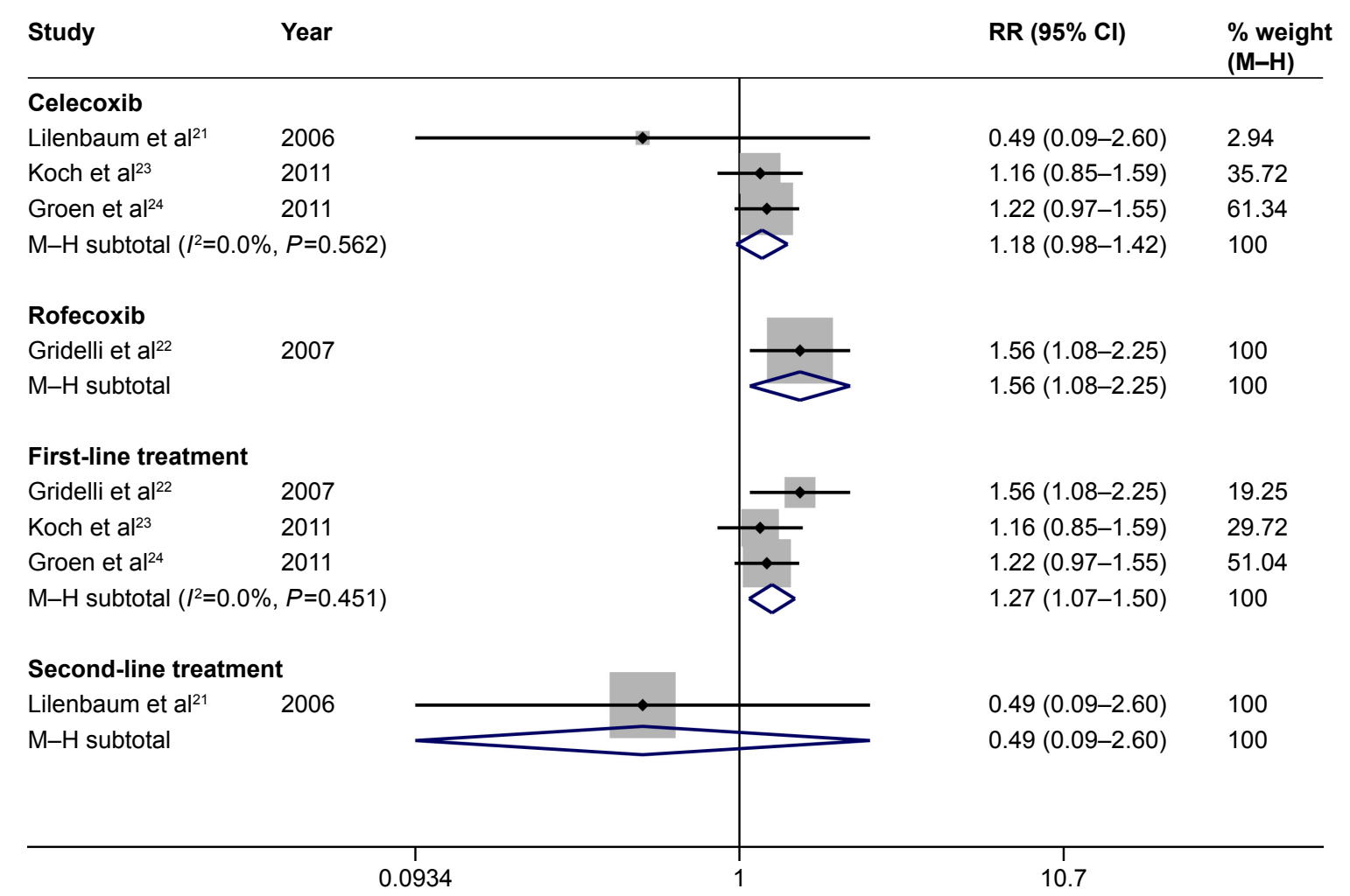

Figure 5 Forest plot of overall response rate from subgroup analysis.

Abbreviations: RR, risk ratio; $\mathrm{M}-\mathrm{H}$, Mantel-Haenszel. 
Table 3 Meta-analysis of the toxicities in patients with advanced NSCLC

\begin{tabular}{llllll}
\hline Toxicity & $\begin{array}{l}\text { Number } \\
\text { of RCTs }\end{array}$ & RR (95\% Cl) & $\begin{array}{l}\text { P-value } \\
\text { for RR }\end{array}$ & $\begin{array}{l}\mathbf{I}^{2} \text { for } \\
\text { heterogeneity }\end{array}$ & $\begin{array}{l}\text { P-value for } \\
\text { heterogeneity }\end{array}$ \\
\hline Leukopenia & 6 & $1.21(1.03-1.42)$ & 0.017 & $0.0 \%$ & 0.499 \\
Thrombocytopenia & 6 & $1.32(1.04-1.67)$ & 0.020 & $0.0 \%$ & 0.56 \\
Anemia & 4 & $1.27(0.71-2.27)$ & 0.416 & $12.0 \%$ & 0.333 \\
Nausea & 4 & $0.70(0.39-1.25)$ & 0.228 & $0.0 \%$ & 0.969 \\
Diarrhea & 3 & $1.31(0.64-2.71)$ & 0.460 & $41.6 \%$ & 0.180 \\
Asthenia & 5 & $0.78(0.50-1.23)$ & 0.289 & $0.0 \%$ & 0.531 \\
Cardiotoxicity & 5 & $2.39(1.06-5.42)$ & 0.037 & $0.0 \%$ & 0.690 \\
\hline
\end{tabular}

Abbreviations: RCTs, randomized controlled trials; NSCLC, non-small-cell lung cancer; RR, risk ratio.

and cardiotoxicity was observed in COX-2 inhibitors plus chemotherapy compared to chemotherapy alone. Few studies $^{31-33}$ reported that coordination of COX-2 with vascular endothelial growth factor (VEGF) may promote angiogenesis in bone marrow after chemotherapy. Pharmacodynamic studies suggested that COX-2 inhibitors can inhibit angiogenesis by inhibiting the VEGF, basic fibroblast growth factor, platelet-derived growth factor, and endothelin $21,{ }^{31}$ which was a possible explanation for a higher frequency of leukopenia and thrombocytopenia in COX-2 inhibitors plus chemotherapy. The induction of cardiovascular events by COX-2 inhibitors limits its applications and research for cancer. Chen et $\mathrm{al}^{12}$ reported that the risk of cardiovascular events was significantly increased in patients with long-term use of celecoxib, whereas the other meta-analyses did not find that COX-2 inhibitors used for treating NSCLC could increase the risk of cardiovascular events. ${ }^{10,11}$ In an attempt to answer the questions about the cardiovascular safety of NSAIDs and COX-2 inhibitors, many physician-scientists have undertaken research efforts. Innumerable observational studies examining larger and larger administrative databases have been sought to answer these critical questions. However, cardiovascular toxicity of COX-2 inhibitors still remains a debated topic in the field.

There are several limitations to this study that should be addressed. First, only a few clinical trials met the inclusion and exclusion criteria. Consequently, more clinical studies will be required to confirm our results. Second, not all RCTs provided sufficient data on response rates and survival indices, which affected the pooled results in the present metaanalysis. Finally, one study had lost large amounts of data, and there may be a certain attrition bias.

\section{Conclusion}

This meta-analysis indicated that COX-2 inhibitors, especially rofecoxib, improved ORR of advanced NSCLC with chemotherapy, but had no effect on survival indices. Accordingly, COX-2 inhibitors may lead to higher rates of hematologic toxicities and cardiovascular events. Based on these findings, benefits versus hazards of COX-2 inhibitors for treating advanced NSCLC need to be carefully considered.

\section{Acknowledgments}

This study was supported by the Project supported by science and technology activities among overseas students and Molecular Genetics and Mechanism of EGFR Gene Mutation in Uygur and Han People with NSCLC in Xinjiang Applying Circulating Tumor DNA Detection (No. 81560381). The funders had no roles in study design, data collection and analysis, decision to publish, or preparation of the manuscript.

\section{Author contributions}

PD and PG had full access to all of the data in the study, and take responsibility for the integrity of the data and the accuracy of the data analysis. PD, XPM, and JH designed the study. JH and JJM developed and tested the data collection forms. PD and XPM acquired the data. XPM and PG conducted the analysis and interpreted the data. XPM and JH drafted the manuscript. All authors contributed toward data analysis, drafting and revising the paper and agree to be accountable for all aspects of the work.

\section{Disclosure}

The authors report no conflicts of interest in this work.

\section{References}

1. Scheff RJ, Schneider BJ. Non-small-cell lung cancer: treatment of late stage disease: chemotherapeutics and new frontiers. Semin Intervent Radiol. 2013;30:191-198.

2. Yokouchi H, Kanazawa K, Ishida T, et al. Cyclooxygenase-2 inhibitors for non-small-cell lung cancer: a phase II trial and literature review. Mol Clin Oncol. 2014;2:744-750.

3. Smith WL, Langenbach R. Why there are two cyclooxygenase isozymes. J Clin Invest. 2001;107:1491-1495.

4. Patel S, Chiplunkar S. Role of cyclooxygenase-2 in tumor progression and immune regulation in lung cancer. Indian J Biochem Biophys. 2007;44:419-428.

5. Sandler AB, Dubinett SM. COX-2 inhibition and lung cancer. Semin Oncol. 2004;31:45-52. 
6. Altorki NK, Port JL, Zhang F, et al. Chemotherapy induces the expression of cyclooxygenase- 2 in non-small cell lung cancer. Clin Cancer Res. 2005;11:4191-4197.

7. Krysan K, Reckamp KL, Sharma S, Dubinett SM. The potential and rationale for COX-2 inhibitors in lung cancer. Anticancer Agents Med Chem. 2006;6:209-220.

8. Nissen SE. Cox-2 inhibitors and cardiovascular disease: considerable heat, but not much light. Eur Heart J. 2012;33:2631-2633.

9. Mukherjee D, Nissen SE, Topol EJ. Risk of cardiovascular events associated with selective COX-2 inhibitors. JAMA. 2001;286: 954-959.

10. Hou LC, Huang F, Xu HB. Does celecoxib improve the efficacy of chemotherapy for advanced non-small cell lung cancer? $\mathrm{Br} \mathrm{J}$ Clin Pharmacol. 2016;81:23-32.

11. Zhou YY, Hu ZG, Zeng FJ, Han J. Clinical profile of cyclooxygenase-2 inhibitors in treating non-small cell lung cancer: a meta-analysis of nine randomized clinical trials. PLoS One. 2016;11:e0151939.

12. Chen J, Shen P, Zhang XC, Zhao MD, Zhang XG, Yang L. Efficacy and safety profile of celecoxib for treating advanced cancers: a meta-analysis of 11 randomized clinical trials. Clin Ther. 2014;36:1253-1263.

13. Moher D, Liberati A, Tetzlaff J, Altman DG. Preferred reporting items for systematic reviews and meta-analyses: the PRISMA statement. BMJ. 2009;339:b2535.

14. Tierney JF, Stewart LA, Ghersi D, Burdett S, Sydes MR. Practical methods for incorporating summary time-to-event data into meta-analysis. Trials. 2007;8:16

15. Higgins JP, Altman DG, Gotzsche PC, et al. The Cochrane Collaboration's tool for assessing risk of bias in randomised trials. BMJ. 2011; 343:d5928.

16. Deeks JJ. Issues in the selection of a summary statistic for metaanalysis of clinical trials with binary outcomes. Stat Med. 2002;21: 1575-1600

17. Higgins JPT, Green S. Cochrane handbook for systematic reviews of interventions, v.5.1; 2011 [Last updated March 5, 2011]. Available from: http://handbook-5-1.cochrane.org. Accessed December 18, 2017.

18. Higgins JP, Thompson SG. Quantifying heterogeneity in a metaanalysis. Stat Med. 2002;21:1539-1558.

19. Copas J, Shi JQ. Meta-analysis, funnel plots and sensitivity analysis. Biostatistics. 2000;1:247-262.

20. Egger M, Davey Smith G, Schneider M, Minder C. Bias in meta-analysis detected by a simple, graphical test. BMJ. 1997;315:629-634.

21. Lilenbaum R, Socinski MA, Altorki NK, et al. Randomized phase II trial of docetaxel/irinotecan and gemcitabine/irinotecan with or without celecoxib in the second-line treatment of non-small-cell lung cancer. J Clin Oncol. 2006;24:4825-4832.
22. Gridelli C, Gallo C, Ceribelli A, et al. Factorial phase III randomised trial of rofecoxib and prolonged constant infusion of gemcitabine in advanced non-small-cell lung cancer: the GEmcitabine-COxib in NSCLC (GECO) study. Lancet Oncol. 2007;8:500-512.

23. Koch A, Bergman B, Holmberg E, et al. Effect of celecoxib on survival in patients with advanced non-small cell lung cancer: a double blind randomised clinical phase III trial (CYCLUS study) by the Swedish Lung Cancer Study Group. Eur J Cancer. 2011;47:1546-1555.

24. Groen HJ, Sietsma H, Vincent A, et al. Randomized, placebo-controlled phase III study of docetaxel plus carboplatin with celecoxib and cyclooxygenase-2 expression as a biomarker for patients with advanced non-small-cell lung cancer: the NVALT-4 study. J Clin Oncol. 2011; 29:4320-4326.

25. Edelman MJ, Tan MT, Fidler MJ, et al. Randomized, double-blind, placebo-controlled, multicenter phase II study of the efficacy and safety of apricoxib in combination with either docetaxel or pemetrexed in patients with biomarker-selected non-small-cell lung cancer. J Clin Oncol. 2015;33:189-194.

26. Edelman MJ, Wang X, Hodgson L, et al. Phase III randomized, placebo-controlled, double-blind trial of celecoxib in addition to standard chemotherapy for advanced non-small-cell lung cancer with cyclooxygenase-2 overexpression: CALGB 30801 (Alliance). J Clin Oncol. 2017;35:2184-2192.

27. Masferrer JL, Leahy KM, Koki AT, et al. Antiangiogenic and antitumor activities of cyclooxygenase-2 inhibitors. Cancer Res. 2000;60: 1306-1311.

28. Laskin JJ, Sandler AB. The importance of the eicosanoid pathway in lung cancer. Lung Cancer. 2003;41(Suppl 1):S73-S79.

29. Hida T, Yatabe $Y$, Achiwa $H$, et al. Increased expression of cyclooxygenase 2 occurs frequently in human lung cancers, specifically in adenocarcinomas. Cancer Res. 1998;58:3761-3764.

30. Trotti A, Colevas AD, Setser A, et al. CTCAE v3.0: development of a comprehensive grading system for the adverse effects of cancer treatment. Semin Radiat Oncol. 2003;13:176-181.

31. Liu XW, He QT, Li ZQ, et al. [Expression of cyclooxygenase-2 in bone marrow cells of leukemia patients and its association with angiogenesis]. Zhongguo Shi Yan Xue Ye Xue Za Zhi. 2009;17:40-42. Chinese.

32. Lorenz M, Slaughter HS, Wescott DM, et al. Cyclooxygenase-2 is essential for normal recovery from 5-fluorouracil-induced myelotoxicity in mice. Exp Hematol. 1999;27:1494-1502.

33. Sanaat Z, Khalili R, Almasi S, et al. Does chemotherapy change expression of VEGF A\&C and MVD in acute myeloid leukemia? Int J Hematol Oncol Stem Cell Res. 2014;8:24-29.
OncoTargets and Therapy

\section{Publish your work in this journal}

OncoTargets and Therapy is an international, peer-reviewed, open access journal focusing on the pathological basis of all cancers, potential targets for therapy and treatment protocols employed to improve the management of cancer patients. The journal also focuses on the impact of management programs and new therapeutic agents and protocols on
Dovepress

patient perspectives such as quality of life, adherence and satisfaction The manuscript management system is completely online and includes a very quick and fair peer-review system, which is all easy to use. Visit http://www.dovepress.com/testimonials.php to read real quotes from published authors. 